\title{
Prevalence and characteristics of intramural coronary artery in hypertrophic obstructive cardiomyopathy: a coronary computed tomography and invasive angiography study
}

\author{
Changsheng Zhu, Shuiyun Wang, Shengwei Wang, Yanhai Meng, Qiulan Yang, Changrong Nie, \\ Hongtao Sun
}

Department of Cardiovascular Surgery, Fuwai Hospital, National Center for Cardiovascular Diseases, Chinese Academy of Medical Sciences and Peking Union Medical College, Beijing, China

Correspondence to: Shuiyun Wang, MD. Department of Cardiovascular Surgery, Fuwai Hospital, National Center for Cardiovascular Diseases, Chinese Academy of Medical Sciences and Peking Union Medical College, Beilishi Road 167, Xicheng District, Beijing 100037, China.

Email: wsymd@sina.com.

\begin{abstract}
Background: The prevalence and morphologic characteristics of intramural coronary artery (ICA) in patients with hypertrophic obstructive cardiomyopathy (HOCM) have yet to be fully illuminated. Our study aimed to investigate the prevalence and morphologic characteristics of ICA in patients with HOCM using coronary computed tomography (CT) angiography and invasive coronary angiography.

Methods: Patients with a diagnosis of HOCM who were admitted for selective myectomy in Fuwai Hospital were prospectively enrolled between September 2015 and June 2019. Both preoperative coronary CT and invasive angiography were scheduled for all participants.

Results: Coronary CT angiography detected ICA in 106 (23.3\%) out of 455 patients. Dynamic compression of coronary arteries was observed in 87 patients $(19.1 \%)$ by invasive coronary angiography. We found ICA covered with complete myocardial encasement in 98 patients $(92.5 \%)$, with deep myocardial bridging $(\mathrm{MB})$ observed most frequently $(\mathrm{P}=0.005)$. All patients with dynamic compression of coronary arteries had ICA. Dynamic luminal reduction $\geq 50 \%$ was present in 77 (16.9\%) of the study participants. Pearson's correlation analysis revealed that the length and degree of dynamic compression were significantly related with MB length and depth (Pearson's correlation r=0.241, 0.581, 0.316, and 0.209; $\mathrm{P}=0.014,<0.001$, 0.002 , and 0.032 , respectively).

Conclusions: Patients with HOCM commonly present with ICA and it can be visualized well by coronary CT angiography. Deep or extensive MB is more likely to produce coronary artery dynamic compression. Preoperative identification of this congenital coronary artery variant may be helpful for surgical planning.
\end{abstract}

Keywords: Hypertrophic obstructive cardiomyopathy (HOCM); intramural coronary artery (ICA); coronary computed tomographic angiography

Submitted Feb 27, 2020. Accepted for publication Aug 06, 2020.

doi: 10.21037/qims-20-362

View this article at: http://dx.doi.org/10.21037/qims-20-362

\section{Introduction}

Hypertrophic cardiomyopathy, the most common monogenic cardiovascular disorder, is characterized by asymmetric septal hypertrophy and variable phenotypic components (1). Myocardial bridging (MB) occurs when the myocardium overlies a segment of the coronary artery that normally has an epicardial course (2). In this situation, the coronary artery running within the $\mathrm{MB}$ is referred to as an intramural coronary artery (ICA). Previous studies have revealed inconsistent but relatively high frequencies 
of $\mathrm{MB}$ in hypertrophic cardiomyopathy, ranging from $40-58 \%$ in children and $11-30 \%$ in adults, as identified by coronary angiography (3-7). Moreover, the reported rates of $\mathrm{MB}$ among hypertrophic cardiomyopathy cases are even higher when detected by coronary computed tomography (CT) and autopsy series, with MB observed in 37 cases of 91 patients in the study by Shariat and colleagues and in 47 cases of 115 patients in the study by Basso and colleagues, respectively $(8,9)$. However, all the previous studies have been either retrospective or of small sample size. As the prevalence of $\mathrm{MB}$ in hypertrophic cardiomyopathy varies widely depending on the imaging modality used, it has yet to be fully established. Therefore, this study aimed to investigate the prevalence and morphologic characteristics of ICA using both coronary CT angiography and invasive coronary angiography and to examine correlations of indices measured by these two imaging modalities in a prospective cohort of consecutive patients with hypertrophic obstructive cardiomyopathy (HOCM).

\section{Methods}

\section{Study population}

Patients with a diagnosis of HOCM who were admitted for selective myectomy in the Department of Cardiovascular Surgery at Fuwai Hospital between September 2015 and June 2019 , were prospectively enrolled in our study. According to the current guidelines, a diagnosis of HOCM was made when the thickness of any segment of the left ventricle was $\geq 15 \mathrm{~mm}$, the patient had no other cardiac or systemic diseases capable of producing a similar magnitude of hypertrophy, and the peak left ventricular outflow tract gradient was $\geq 30 \mathrm{mmHg}$ $(10,11)$. Both preoperative coronary CT and invasive angiography were scheduled, and the patients' clinical data were prospectively collected. The study was approved by the Institutional Ethics Committee of Fuwai Hospital and written informed consent was given by all participants.

\section{Coronary CT angiography}

Coronary CT angiography was performed with a 64-row LightSpeed VCT scanner (GE Healthcare, Milwaukee, WI, USA) as previously described (12). Patients with a heart rate of $>70$ beats $/ \mathrm{min}$ (bpm) were orally administered $25-50 \mathrm{mg}$ of metoprolol (Selokeen; AstraZeneca, Zoetermeer, The Netherlands) before scanning. Scanning was performed at $120 \mathrm{kV}$ and $600-1,000 \mathrm{~mA}$, with a detector collimation of $0.625 \mathrm{~mm}$, a gantry rotation time of $0.35 \mathrm{~s}$, and a pitch of $0.16-0.22$. Tube current ranged from $200-550 \mathrm{~mA}$ and was modulated according to electrocardiogram (ECG) [the tube current was $550 \mathrm{~mA}$ for $40-80 \%$ of an interbeat (RR) interval where diagnostic image quality was required and $200 \mathrm{~mA}$ during the other phase of the RR interval]. According to the patients' weight and time to aortic peak enhancement, an appropriate volume of contrast media was injected into an antecubital vein at a rate of $5 \mathrm{ml} / \mathrm{s}$ through a 20-gauge trocar using a dual-head power injector (Stellant, Medrad, Pittsburgh, PA, USA). Image reconstruction was routinely performed at $75 \%$ of the RR interval. If motion artifacts were apparent, the image was additionally reconstructed at $40 \%, 50 \%, 60 \%, 70 \%$, and $80 \%$ of the RR interval, and then the best quality reconstructed images quality were chosen. Thereafter, images were transferred to a standalone workstation (Deep Blue, ADW4.3, GE Healthcare, Milwaukee, WI, USA) and evaluated by an experienced radiologist using dedicated software (Vitrea FX 1.0, Vital Images, Minnetonka, Minnesota).

Axial and cross-sectional views, as well as multiplanar reformation images, were used to evaluate ICA (13-15). A diagnosis of ICA was made when a segment of the coronary artery was either covered by the myocardium, or located within the interventricular groove and in direct contact with myocardium but not fully encased by a visible layer of myocardium or fibrous-fatty tissue (Figure 1). Thus, the ICA was classified as complete encasement with myocardium or partial encasement with myocardium correspondingly. The former was further classified as follows: superficial pattern: an overlying myocardial layer of $<2 \mathrm{~mm}$; deep pattern: an overlying myocardial layer of between $\geq 2 \mathrm{~mm}$ and $<5 \mathrm{~mm}$; and very deep pattern: an overlying myocardial layer of $\geq 5 \mathrm{~mm}$. For each tunneled segment of the ICA, the anatomical parameters measured were as follows: (I) involved location and segment of the intramural course; (II) the length, depth (maximal thickness of the myocardial layer from the epicardial surface), and diameter of the intramural segment; and (III) the diameter of the coronary artery at the intramural entry. The ICA was further classified as extensive if the length of the intramural course was $\geq 25 \mathrm{~mm}$.

\section{Invasive coronary angiography}

Invasive coronary angiography was performed using standard techniques, with at least two different views for each main coronary artery through a femoral or radial approach. Images were reviewed by an experienced 

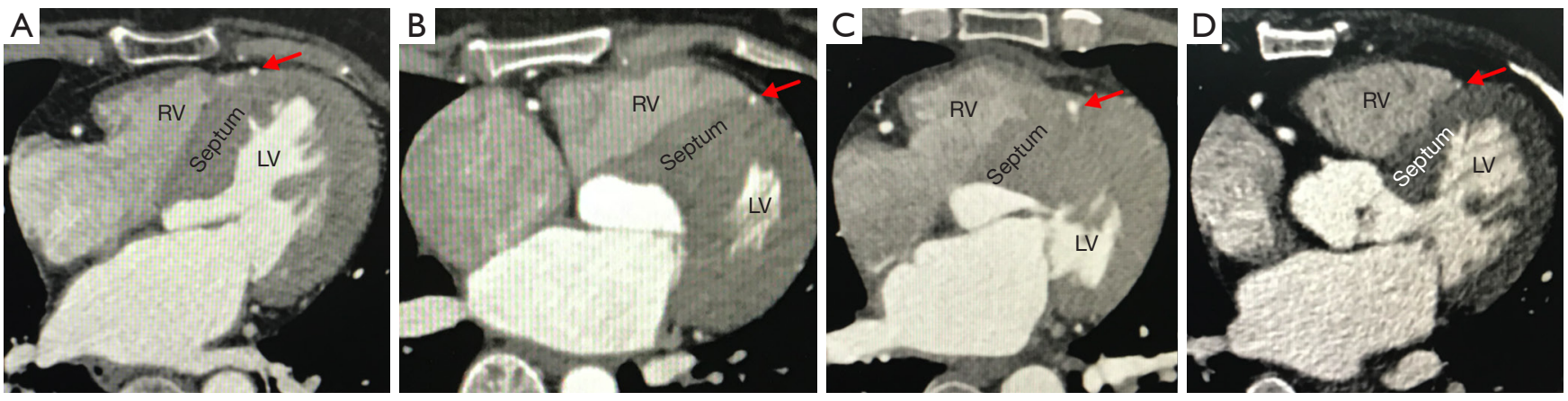

Figure 1 Different morphological types of intramural coronary artery in cross sectional view. (A) Coronary computed tomography angiography axial plane view of ICA with partial myocardial encasement, (B,C) complete encasement by superficial and deep MB (D) penetrating into the right ventricular intracavity. Red arrows indicate intramural coronary artery in cross section. CT, computed tomography; ICA, intramural coronary artery; MB, myocardial bridging; RV, right ventricle; LV, left ventricle.

radiologist blinded to the results of coronary CT angiography. Angiographic diagnosis of ICA was made when dynamic compression of an epicardial coronary artery was present. For each ICA, the following parameters were measured: (I) the degree and total length of the dynamic compression during systole; and (II) the diameter of the compressed coronary artery at both systole and diastole. Dynamic luminal reductions were defined as follows: $<50 \%$ (mild); $\geq 50 \%$ and $<70 \%$ (moderate); $\geq 70 \%$ and $<90 \%$ (severe); and $\geq 90 \%$ (extremely severe).

\section{Statistical analysis}

Categorical variables are presented as frequency and percentage, and were compared using the chi-squared $\left(\chi^{2}\right)$ or Fisher's exact test. Continuous variables are presented as mean \pm standard deviation or median, and were compared using the two-sample $t$-test where appropriate. Pearson's correlation analysis was used to examine the relationships between continuous variables. Statistical significance was set as a two-tailed $\mathrm{P}$ value of $\leq 0.05$. All statistical analyses were performed using SPSS version 22.0 (SPSS Inc., IBM Company, Chicago, IL, USA).

\section{Results}

\section{Patient characteristics and prevalence of MB in HOCM}

Between September 2015 and June 2019, 649 consecutive patients with HOCM were admitted to the Department of Cardiovascular Surgery at Fuwai Hospital for selective septal myectomy. Among them, 187 patients were excluded from the study due to having undertaken coronary CT angiography or invasive angiography at other hospitals prior to admission. Therefore, 462 patients were prospectively included into the present study and subsequent coronary CT angiography and invasive angiography were scheduled (Figure 2). Due to inadequate image quality, 7 patients were excluded from the final analysis. The baseline characteristics of patients excluded from and included in our study are listed in Table S1.

Patient characteristics are presented in Table 1. The present study comprised 455 patients with HOCM, of whom $174(38.0 \%)$ were female. The mean age of the patients was $45.8 \pm 14.7$ (range, 8.0-71.0) years. ICA was observed in 106 (23.3\%) out of 455 patients on coronary CT angiography. Dynamic compression of the coronary artery was detected in 87 patients $(19.1 \%)$ by invasive coronary angiography (Figure 2). Patients with ICA were younger $(41.2 \pm 14.8$ vs. $47.2 \pm 14.4$ years, $\mathrm{P}<0.001)$, more likely to have a family history of hypertrophic cardiomyopathy (HCM; $17.3 \% v s$. $8.1 \%, \mathrm{P}=0.011)$, and had a lower rate of hypertension $(19.1 \%$ vs. $31.1 \%, \mathrm{P}=0.015)$ than patients without ICA.

\section{Anatomic characteristics of MB in HOCM}

Detailed characteristics of ICA and MB detected by coronary $\mathrm{CT}$ and invasive angiography are presented in Table 2.

\section{Coronary CT angiography}

A total of 117 intramural segments were found in 106 patients with ICA. Multiple intramural segments were observed in 3 patients $(2.8 \%)$. The most frequently involved 


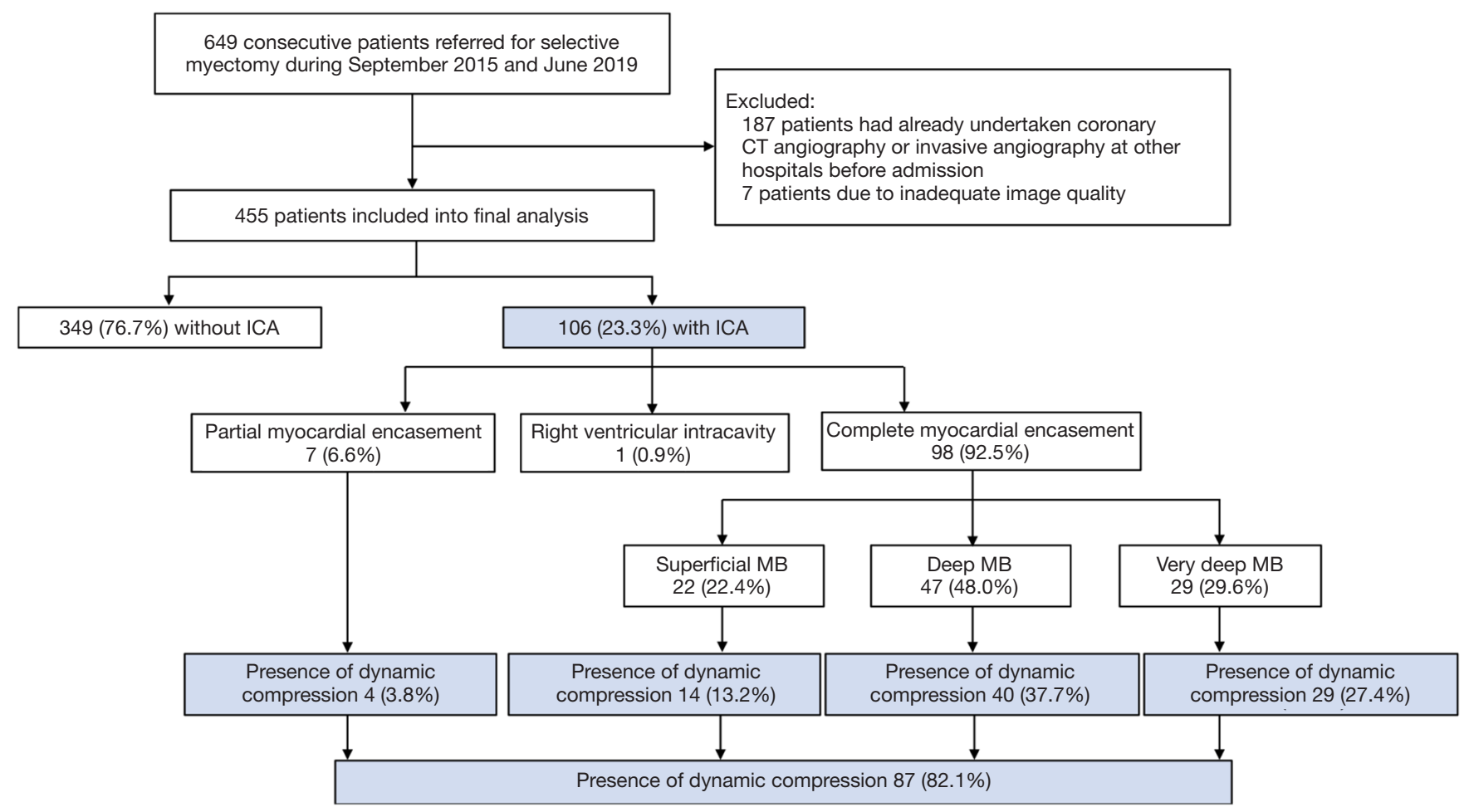

Figure 2 Flowchart of the study population. ICA, intramural coronary artery; MB, myocardial bridging.

segment was the middle of the left anterior descending coronary artery, which was observed in 89 patients (86.4\%). Notably, 1 patient $(1.0 \%)$ had an ICA that penetrated the right ventricular intracavity (Figure 1D). No ICAs were found in the right coronary arteries of the patients.

Among the 106 patients with ICA, complete myocardial encasement was found in 98 patients $(92.5 \%)$, with deep MB most frequently observed $(\mathrm{P}=0.005)$. The mean maximal depth of $\mathrm{MB}$ was $3.8 \pm 2.8 \mathrm{~mm}$ and the mean value of $M B$ length was $37.2 \pm 21.9 \mathrm{~mm}$, with extensive $\mathrm{MB}$ most frequently detected $(\mathrm{P}<0.001)$. The mean diameter of the ICAs was smaller than that of the coronary arteries at the intramural entrance $(2.9 \pm 0.9$ vs. $3.7 \pm 1.0 \mathrm{~mm}, \mathrm{P}<0.001)$.

\section{Invasive coronary angiography}

All patients with dynamic compression of the coronary artery had ICA (Figure 3). Among 87 patients with coronary dynamic compression, 77 (88.5\%) had a dynamic luminal reduction of $\geq 50 \%$, accounting for $16.9 \%$ of the entire study population. Moreover, almost one-third had a dynamic luminal reduction of $\geq 90 \%$. Notably, 4 patients with partial myocardial encasement had coronary dynamic compression (Figure 3A). The mean percentage and length of dynamic compression were $54.4 \pm 32.3$ and $22.2 \pm$ $14.9 \mathrm{~mm}$, respectively. At end-diastole and end-systole, the ICA diameter was $3.7 \pm 1.1$ and $2.9 \pm 0.8 \mathrm{~mm}$, respectively. Consistent with our observation on coronary CT angiography, the diameter of the ICAs at end-diastole was smaller than that of the coronary arteries at the intramural entrance $(2.9 \pm 0.8$ vs. $3.7 \pm 1.1 \mathrm{~mm}, \mathrm{P}<0.001)$.

\section{Correlation of clinical findings}

Compared to patients without ICA, patients with ICA had a thicker interventricular septum $(20.2 \pm 4.9$ vs. $19.0 \pm 4.3 \mathrm{~mm}$, $\mathrm{P}=0.026$ ) overall (Table 1). However, after propensity scoring matching for age, body mass index, presence of hypertension, and family history of HCM, interventricular septal thickness was similar between the two groups (Table S2). Neither left ventricular (LV) outflow gradient nor documented non-sustained ventricular tachycardia on ambulatory ECG was associated with the presence of ICA.

In patients with coronary dynamic compression, the length of dynamic compression measured using invasive coronary angiography was smaller than that of ICA 
Table 1 Patient characteristics in the present study

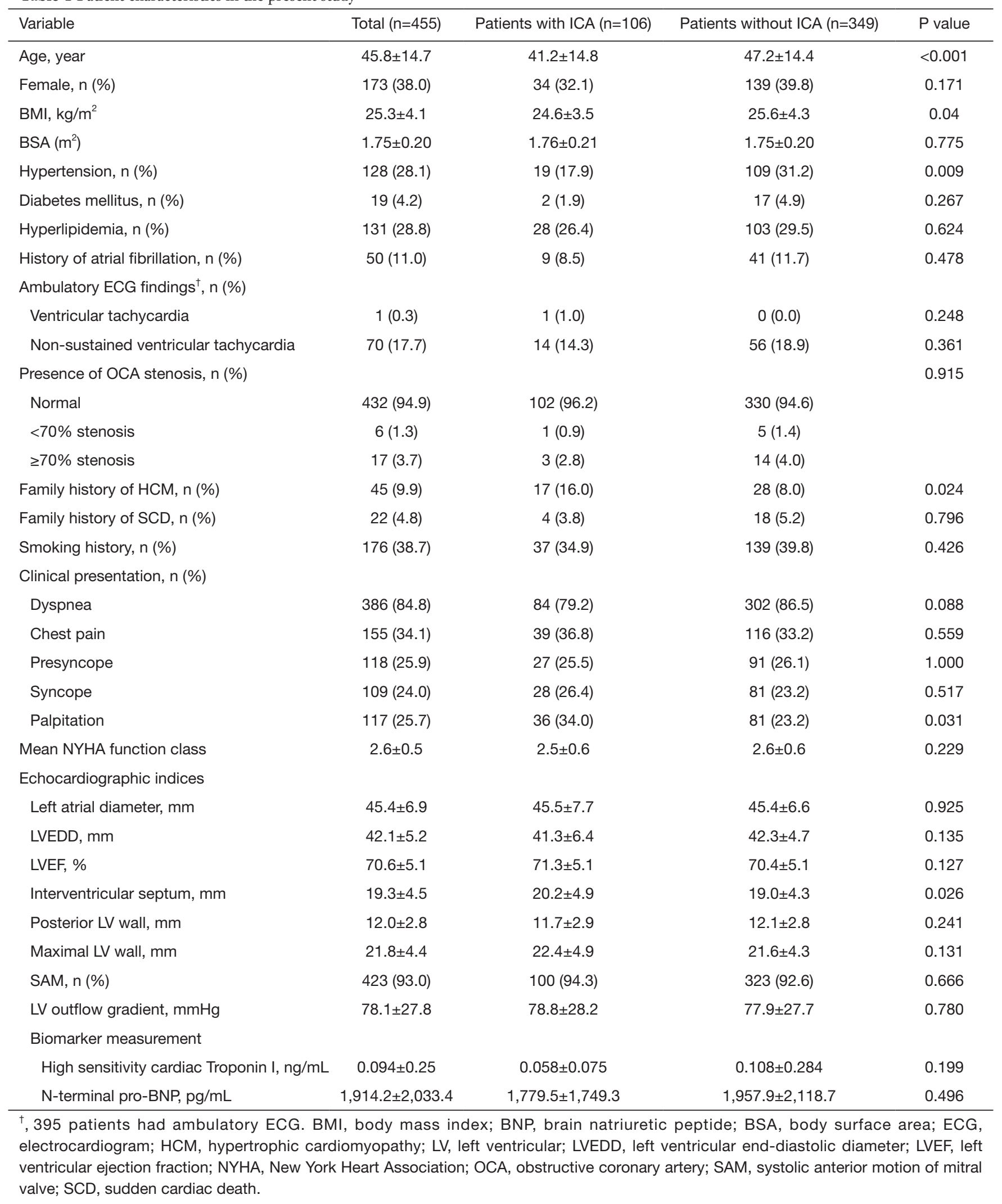


Table 2 Characteristics of intramural coronary artery (ICA) and myocardial bridging (MB) on coronary computed tomography (CT) and invasive angiography

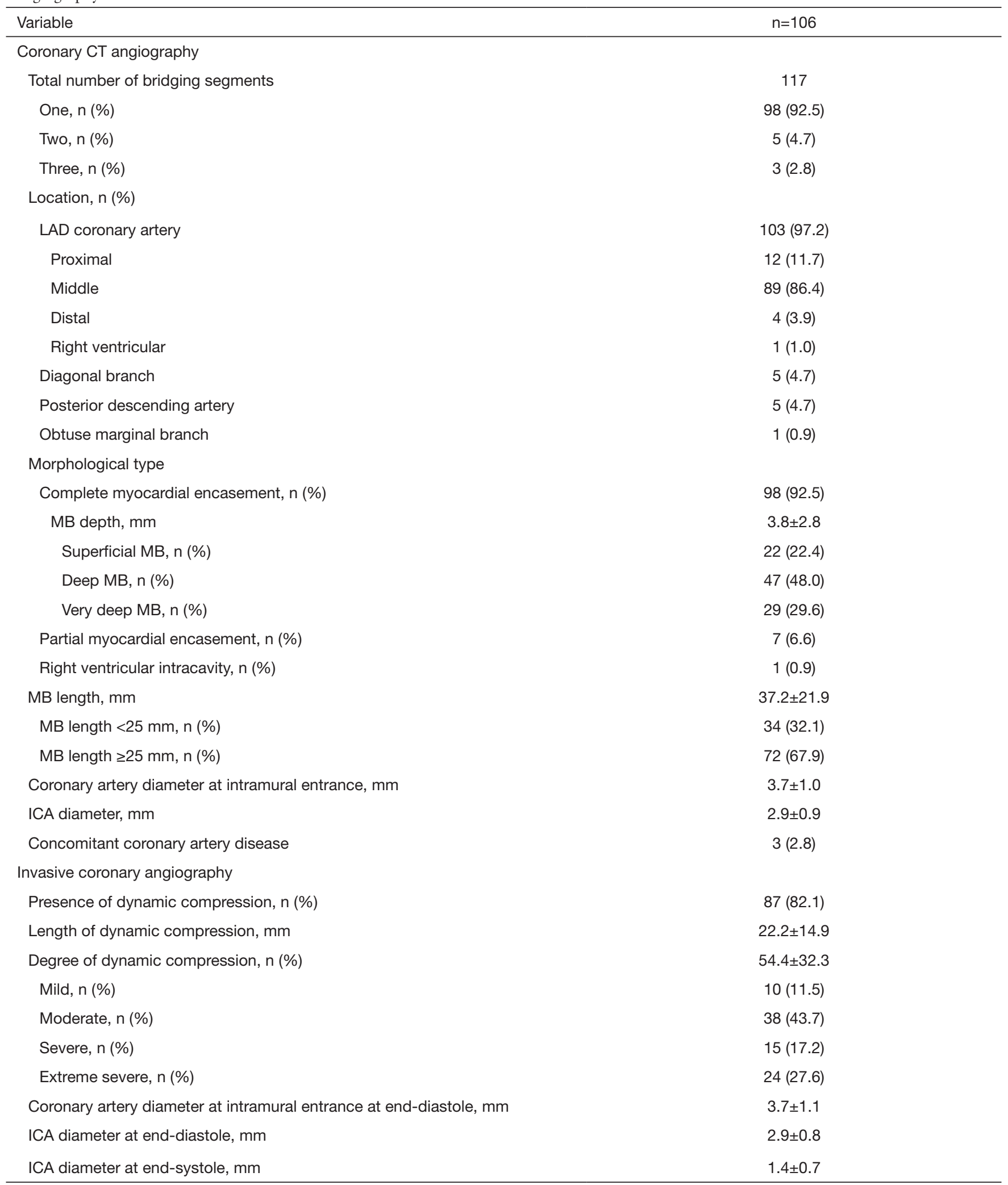

ICA, intramural coronary artery; LAD, left anterior descending artery; MB, myocardial bridging. 

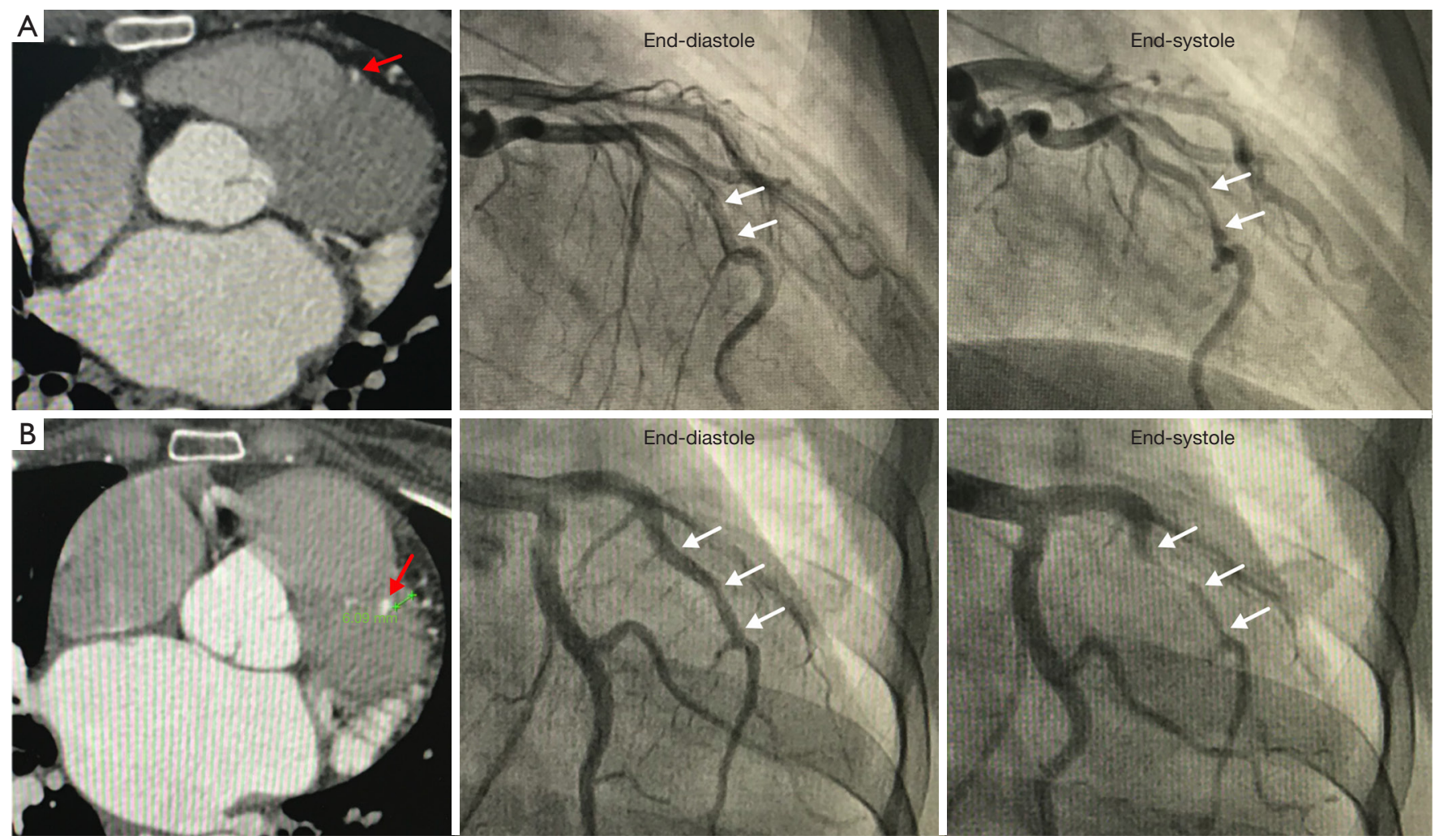

Figure 3 Coronary CT angiography demonstrating ICA with dynamic compression on invasive coronary angiography (A) partially encased by myocardium (B) deeply encased by myocardium. Red arrows indicate intramural coronary artery in cross section. White arrows indicate intramural coronary artery at end-diastole and end-systole from left to right, respectively. CT, computed tomography; ICA, intramural coronary artery.

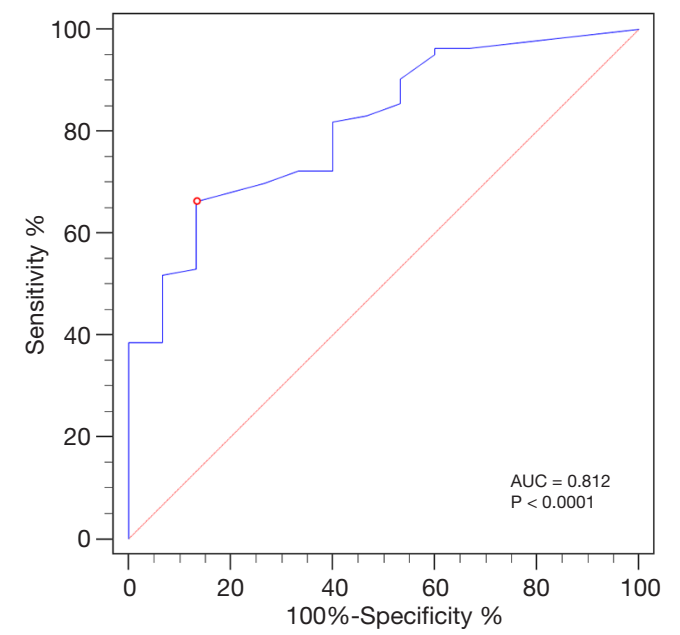

Figure 4 Receiver operating characteristic curve. Myocardial bridging with a depth of $\geq 3 \mathrm{~mm}$ could produce coronary compression. Red circle indicates the best cut-off value of $3 \mathrm{~mm}$. measured using coronary CT angiography $(22.2 \pm 14.9$ vs. $39.0 \pm 23.0 \mathrm{~mm}, \mathrm{P}<0.001)$. Pearson's correlation analysis further revealed that the length of dynamic compression was significantly related with $M B$ depth and length (Pearson's correlation $\mathrm{r}=0.241$ and $0.581, \mathrm{P}=0.014$ and $<0.001$, respectively). Likewise, a significant relationship was found between the degree of dynamic compression and $\mathrm{MB}$ depth and length on Pearson's correlation analysis (Pearson's correlation $\mathrm{r}=0.316$ and $0.209, \mathrm{P}=0.002$ and 0.032 , respectively). Further receiver operating characteristic (ROC) curve analysis (Figure 4) identified a depth of $3 \mathrm{~mm}$ as the cut-off value leading to coronary compression (area under curve $=0.812,95 \%$ confidence interval: $0.720-0.884$, $\mathrm{P}<0.0001)$.

\section{Discussion}

The present study was the first prospective cohort study to 
investigate the prevalence and morphologic characteristics of ICA using coronary CT and invasive angiography in patients with HOCM. We firstly found that the prevalence of ICA detected in the present study was $23.3 \%$ with coronary CT angiography, whereas the rate of coronary dynamic compression detected by invasive coronary angiography was $19.1 \%$. Second, most of the ICAs (98/106, $92.5 \%$ ) were encircled by a complete layer of MB. Deep or extensive MB was common in patients with HOCM. Third, most patients with ICA $(87 / 106,82.1 \%)$ had a dynamic luminal reduction of $\geq 50 \%$. Fourth, the depth and length of the ICA were related to the degree and length of dynamic compression. Lastly, MB was not related to interventricular septal thickness after adjustment.

Both ICA or MB are relatively common findings with the increasing use of coronary CT angiography, especially in patients with chest pain $(13,16)$. Previous studies have indicated that $\mathrm{MB}$ was more common in cardiac diseases that are associated with left ventricular hypertrophy, such as aortic stenosis or $\operatorname{HCM}(3,17)$. However, studies investigating $\mathrm{MB}$ in patients with hypertrophic cardiomyopathy are lacking. Related studies have been limited to small series in the earlier time or retrospective case series of conventional angiography and autopsy $(3,6,8,18)$. The prevalence of ICA detected by coronary CT angiography in the present study was $23.3 \%$ among patients with HOCM, which is close to the prevalence of $22 \%$ reported in a recent study of coronary CT evaluating patients with a low-to-intermediate pre-test probability and without obstructive coronary artery disease (16). Therefore, the prevalence of ICA among patients with HOCM may be similar to that in the general population rather than higher, as previously suggested. In our study, coronary dynamic compression was detected in $19.1 \%$ of the study population, which is lower than the rate of ICA detected using coronary CT angiography in previous reports $(17,19)$.

Apart from differences in the imaging modalities used, variable definitions of $\mathrm{MB}$ or ICA have also contributed to variability in the reported rates of previous studies. From our point of view, although MB and ICA actually depict the same phenomenon of coronary artery variation, they are not completely equivalent in different reports. The definition of MB only includes ICA with complete myocardial encasement; thus, an underestimated prevalence is expected. In accordance with our observations and those of previous reports, a minority of ICAs with partial myocardial encasement could also produce dynamic compression of involved vessel segments, which have usually been overlooked (13-15). Hence, the term "ICA" may be more appropriate to cover this phenomenon concerning a congenital coronary artery variant.

Invasive coronary angiography identifies ICA indirectly by detecting the presence of milking effect, which belongs to function evaluation (17). Nevertheless, MB and ICA convey an anatomic conception. Not all instances of $\mathrm{MB}$ or ICA involve the capability of vessel compression. Therefore, it seems reasonable that the detection rates of $\mathrm{MB}$ by invasive coronary angiography usually rank last $(17,19)$. In line with our observations, deep or extensive MB is more likely to produce dynamic compression.

\section{Correlations of findings on coronary $C T$ and invasive angiography}

In our study focusing on patients with HOCM, most examples of ICA had complete myocardial encasement, among which the extent of MB was predominantly deep or long. The corresponding manifestation on invasive coronary angiography was that most ICAs had a dynamic luminal reduction of $\geq 50 \%$. Further, Pearson's correlation analysis suggested relationships between the depth and length of the ICA on coronary CT angiography and the degree and length of dynamic compression on invasive coronary angiography. Consistent with Kim et al.'s study, the length of the ICA on coronary CT angiography was significantly related to dynamic compression (14). Contrary to this previous study, the length of ICA measured on coronary CT angiography was longer than the length of dynamic compression measured on invasive coronary angiography in our study. Additionally, in our study, both the depth and length of $\mathrm{MB}$ were related with the degree of dynamic compression in all ICAs with complete myocardial encasement. However, these findings explain the following observations. First, dynamic compression can occur in an ICA with partial myocardial encasement and without overlying myocardium directly above it. We speculate that if the ICA runs within the interventricular groove in a long enough course, the bilateral ventricular walls of the interventricular groove may squeeze the coronary artery therein, resulting in dynamic compression, regardless of the presence of overlying myocardium. Second, deep MB is more likely to produce dynamic compression, whereas superficial MB is less likely to or cannot produce dynamic compression. This also explains the observation that in the same segment of an ICA, the degree of dynamic compression varies with the depth of the overlying 
myocardium, as the epicardial coronary artery usually runs gradually into the myocardium, resulting in varied depths at different sites of the intramural course. Despite the coronary arteries being fully encircled by $\mathrm{MB}$, the thickness of $\mathrm{MB}$ is likely not consistent all the way around.

\section{Clinical relevance concerning ICA and $M B$}

Although the impacts of ICA and MB on the clinical prognosis of HCM in previous studies have been inconsistent, the identification of an ICA or MB may be helpful for myectomy surgeons $(6,8,18)$. Coronary CT suitably visualizes the location and intramural course of an ICA within the interventricular septum, which may help the surgeon to avoid injuring the coronary artery when performing septal myectomy. As surgical unroofing of $\mathrm{MB}$ has been proven safe, preoperative acquaintance with $\mathrm{MB}$ may help the surgeon with individualized surgical planning for relevant HOCM patients (20).

\section{Study limitations}

The present study has some limitations. First, due to the study having been conducted in a dedicated hypertrophic cardiomyopathy center with prospectively included HOCM patients referred for selective myectomy surgery, the prevalence of ICA may have been higher than in the general HCM population. Thus, we recommend that our findings be interpreted with caution. Second, this was a descriptive study aiming to comprehensively depict the morphologic characteristics of ICA and $\mathrm{MB}$ on coronary $\mathrm{CT}$ and conventional invasive angiography, and to compare the related indices measured by these two imaging modalities and identify their correlations. Third, as patients were admitted to our department for scheduled surgery, prognosis analysis regarding the natural clinical course according to the presence of an ICA was not applicable. Therefore, further prospective research is warranted to refine the impacts of different types of ICA on the prognosis of patients with HOCM.

\section{Conclusions}

In conclusion, ICA is common in patients with HOCM and can be visualized well by coronary CT. Deep or extensive $\mathrm{MB}$ is more likely produce dynamic compression. Preoperative identification of this congenital coronary artery variant may be helpful for surgical planning.

\section{Acknowledgments}

Funding: This work was supported by the National Natural Science Foundation of China (Grant No. 81570276) and the Beijing Science and Technology Program of China (Z161100000516154).

\section{Footnote}

Conflicts of Interest: All authors have completed the ICMJE uniform disclosure form (available at http://dx.doi. org/10.21037/qims-20-362). SW reports grants from National Natural Science Foundation of China, grants from Beijing Science and Technology Program of China, during the conduct of the study. The other authors have no conflicts of interest to declare.

Ethical Statement: The hospital ethics committee approved this study, and written informed consent was obtained from each participant (or their guardian).

Open Access Statement: This is an Open Access article distributed in accordance with the Creative Commons Attribution-NonCommercial-NoDerivs 4.0 International License (CC BY-NC-ND 4.0), which permits the noncommercial replication and distribution of the article with the strict proviso that no changes or edits are made and the original work is properly cited (including links to both the formal publication through the relevant DOI and the license). See: https://creativecommons.org/licenses/by-nc-nd/4.0/.

\section{References}

1. Maron BJ. Clinical Course and Management of Hypertrophic Cardiomyopathy. N Engl J Med 2018;379:655-68.

2. Tarantini G, Cademartiri F. Myocardial bridging and prognosis: more evidence but jury still out. Eur Heart J Cardiovasc Imaging 2013;14:515-7.

3. Mohiddin SA, Begley D, Shih J, Fananapazir L. Myocardial bridging does not predict sudden death in children with hypertrophic cardiomyopathy but is associated with more severe cardiac disease. J Am Coll Cardiol 2000;36:2270-8.

4. Yetman AT, Hamilton RM, Benson LN, McCrindle BW. Long-term outcome and prognostic determinants in children with hypertrophic cardiomyopathy. J Am Coll Cardiol 1998;32:1943-50. 
5. Tian T, Wang YL, Wang JZ, Sun K, Zou YB, Zhang WL, Zhu L, Shen H, Hui RT, Zhou XL, Song L. Myocardial bridging as a common phenotype of hypertrophic cardiomyopathy has no effect on prognosis. Am J Med Sci 2014:347:429-33.

6. Sorajja P, Ommen SR, Nishimura RA, Gersh BJ, Tajik AJ, Holmes DR. Myocardial bridging in adult patients with hypertrophic cardiomyopathy. J Am Coll Cardiol 2003;42:889-94.

7. Kitazume H, Kramer JR, Krauthamer D, El Tobgi S, Proudfit WL, Sones FM. Myocardial bridges in obstructive hypertrophic cardiomyopathy. Am Heart J 1983;106:131-5.

8. Basso C, Thiene G, Mackey-Bojack S, Frigo AC, Corrado D, Maron BJ. Myocardial bridging, a frequent component of the hypertrophic cardiomyopathy phenotype, lacks systematic association with sudden cardiac death. Eur Heart J 2009;30:1627-34.

9. Shariat $M$, Thavendiranathan $\mathrm{P}, \mathrm{Nguyen} \mathrm{E}$, Wintersperger B, Paul N, Rakowski H, Crean AM. Utility of coronary CT angiography in outpatients with hypertrophic cardiomyopathy presenting with angina symptoms. J Cardiovasc Comput Tomogr 2014;8:429-37.

10. Elliott PM, Anastasakis A, Borger MA, Borggrefe M, Cecchi F, Charron P, Hagege AA, Lafont A, Limongelli G, Mahrholdt H, McKenna WJ, Mogensen J, Nihoyannopoulos P, Nistri S, Pieper PG, Pieske B, Rapezzi C, Rutten FH, Tillmanns C, Watkins H. 2014 ESC Guidelines on diagnosis and management of hypertrophic cardiomyopathy: the Task Force for the Diagnosis and Management of Hypertrophic Cardiomyopathy of the European Society of Cardiology (ESC). Eur Heart J 2014;35:2733-79.

11. Gersh BJ, Maron BJ, Bonow RO, Dearani JA, Fifer MA, Link MS, Naidu SS, Nishimura RA, Ommen SR, Rakowski H, Seidman CE, Towbin JA, Udelson JE, Yancy CW. 2011 ACCF/AHA Guideline for the Diagnosis and Treatment of Hypertrophic Cardiomyopathy: Executive Summary: A Report of the American College of Cardiology Foundation/American Heart Association Task Force on Practice Guidelines. Circulation 2011;124:2761-96.

12. Lu JG, Lv B, Chen XB, Tang X, Jiang SL, Dai RP. What is the best contrast injection protocol for 64-row multidetector cardiac computed tomography? Eur J Radiol 2010;75:159-65.
13. Rubinshtein R, Gaspar T, Lewis BS, Prasad A, Peled N, Halon DA. Long-term prognosis and outcome in patients with a chest pain syndrome and myocardial bridging: a 64-slice coronary computed tomography angiography study. Eur Heart J Cardiovasc Imaging 2013;14:579-85.

14. Kim PJ, Hur G, Kim SY, Namgung J, Hong SW, Kim YH, Lee WR. Frequency of myocardial bridges and dynamic compression of epicardial coronary arteries: a comparison between computed tomography and invasive coronary angiography. Circulation 2009;119:1408-16.

15. Konen E, Goitein O, Sternik L, Eshet Y, Shemesh J, Di Segni E. The prevalence and anatomical patterns of intramuscular coronary arteries: a coronary computed tomography angiographic study. J Am Coll Cardiol 2007;49:587-93.

16. Dimitriu-Leen AC, van Rosendael AR, Smit JM, van Elst T, van Geloven N, Maaniitty T, Jukema JW, Delgado V, Scholte A, Saraste A, Knuuti J, Bax JJ. LongTerm Prognosis of Patients With Intramural Course of Coronary Arteries Assessed With CT Angiography. JACC Cardiovasc Imaging 2017;10:1451-8.

17. Tarantini G, Migliore F, Cademartiri F, Fraccaro C, Iliceto S. Left Anterior Descending Artery Myocardial Bridging: A Clinical Approach. J Am Coll Cardiol 2016;68:2887-99.

18. Yetman AT, McCrindle BW, MacDonald C, Freedom RM, Gow R. Myocardial bridging in children with hypertrophic cardiomyopathy--a risk factor for sudden death. $\mathrm{N} \mathrm{Engl} \mathrm{J}$ Med 1998;339:1201-9.

19. Corban MT, Hung OY, Eshtehardi P, Rasoul-Arzrumly E, McDaniel M, Mekonnen G, Timmins LH, Lutz J, Guyton RA, Samady H. Myocardial bridging: contemporary understanding of pathophysiology with implications for diagnostic and therapeutic strategies. J Am Coll Cardiol 2014;63:2346-55.

20. Kunkala MR, Schaff HV, Burkhart H, Sandhu GS, Spoon DB, Ommen SR, Sorajja P, Dearani JA. Outcome of repair of myocardial bridging at the time of septal myectomy. Ann Thorac Surg 2014;97:118-23.

Cite this article as: Zhu C, Wang S, Wang S, Meng Y, Yang Q, Nie C, Sun H. Prevalence and characteristics of intramural coronary artery in hypertrophic obstructive cardiomyopathy: a coronary computed tomography and invasive angiography study. Quant Imaging Med Surg 2021;11(1):162-171. doi: 10.21037/qims-20-362 
Supplementary

Table S1 Characteristics of patients included in and excluded from the present study

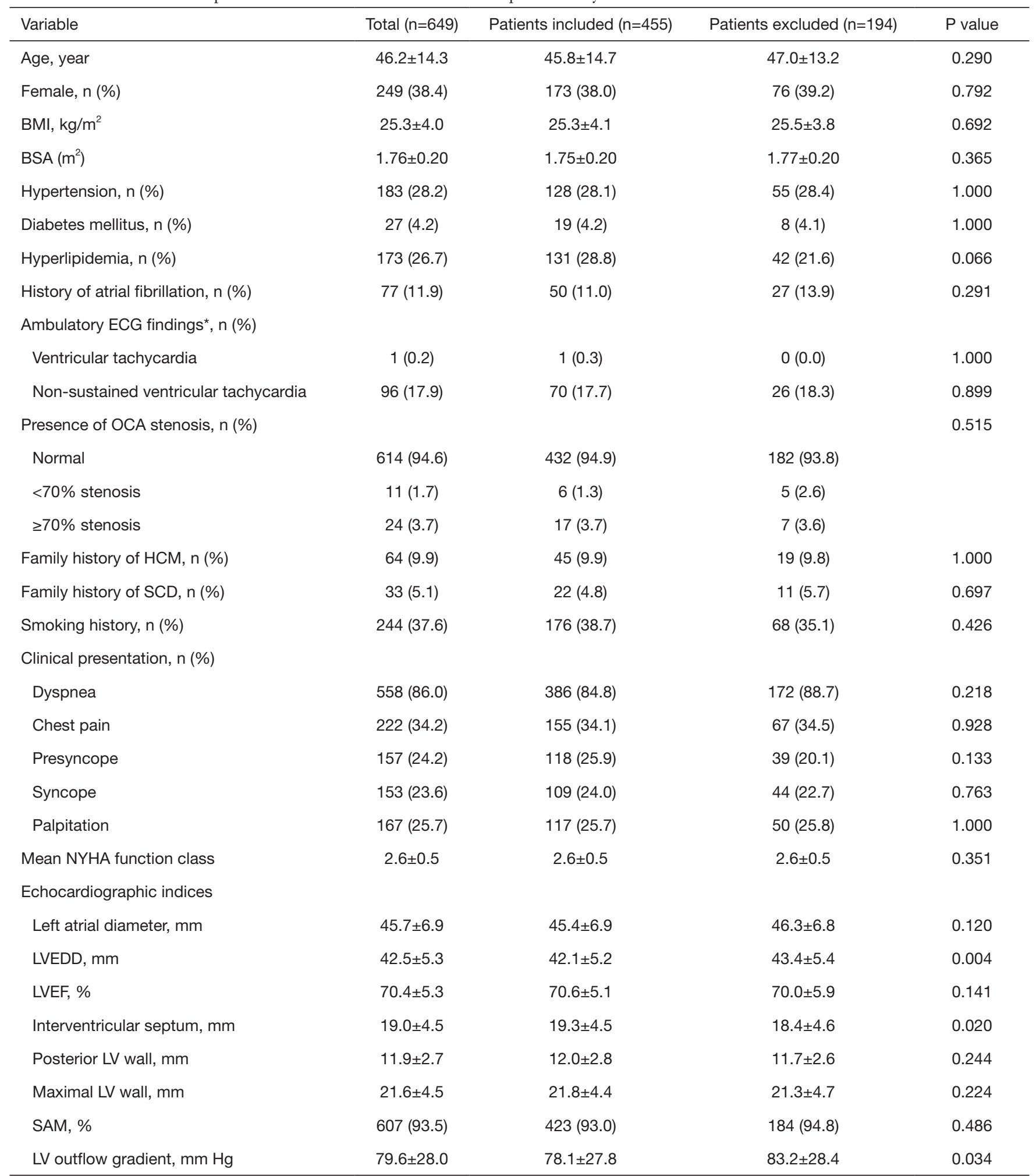

*, 537 patients had ambulatory ECG. BMI, body mass index; BSA, body surface area; ECG, electrocardiogram; HCM, hypertrophic cardiomyopathy; LV, left ventricular; LVEDD, left ventricular end-diastolic diameter; LVEF, left ventricular ejection fraction; NYHA, New York Heart Association; OCA, obstructive coronary artery; SAM, systolic anterior motion of mitral valve; SCD, sudden cardiac death. 
Table S2 Comparison of characteristics among patients with and without intramural coronary artery in the matched cohort

\begin{tabular}{|c|c|c|c|}
\hline Variable & Patients with ICA $(n=106)$ & Patients without ICA $(n=106)$ & $P$ value \\
\hline Female, n (\%) & $34(32.1)$ & $41(38.7)$ & 0.389 \\
\hline BMI, $\mathrm{kg} / \mathrm{m}^{2}$ & $24.6 \pm 3.5$ & $25.1 \pm 3.5$ & 0.347 \\
\hline $\mathrm{BSA}\left(\mathrm{m}^{2}\right)$ & $1.76 \pm 0.21$ & $1.75 \pm 0.21$ & 0.796 \\
\hline Diabetes mellitus, n (\%) & $2(1.9)$ & $4(3.8)$ & 0.683 \\
\hline Hyperlipidemia, n (\%) & $28(26.4)$ & $29(27.4)$ & 1.000 \\
\hline History of atrial fibrillation, $\mathrm{n}(\%)$ & $9(8.5)$ & $5(4.7)$ & 0.408 \\
\hline \multicolumn{4}{|l|}{ Ambulatory ECG findings ${ }^{\dagger}, \mathrm{n}(\%)$} \\
\hline Presence of OCA stenosis, n (\%) & & & 1.000 \\
\hline Normal & $102(96.2)$ & $103(97.2)$ & \\
\hline$<70 \%$ stenosis & $1(0.9)$ & $1(0.9)$ & \\
\hline$\geq 70 \%$ stenosis & $3(2.8)$ & $2(1.9)$ & \\
\hline Family history of HCM, n (\%) & $17(16.0)$ & $11(10.4)$ & 0.310 \\
\hline Family history of SCD, n (\%) & $4(3.8)$ & $8(7.5)$ & 0.374 \\
\hline Smoking history, n (\%) & 37 (34.9) & 37 (34.9) & 1.000 \\
\hline \multicolumn{4}{|l|}{ Clinical presentation, n (\%) } \\
\hline Mean NYHA function class & $2.5 \pm 0.6$ & $2.6 \pm 0.5$ & 0.573 \\
\hline \multicolumn{4}{|l|}{ Echocardiographic indices } \\
\hline Left atrial diameter, $\mathrm{mm}$ & $45.5 \pm 7.7$ & $44.5 \pm 7.0$ & 0.357 \\
\hline LVEDD, mm & $41.3 \pm 6.4$ & $41.8 \pm 5.0$ & 0.536 \\
\hline LVEF, \% & $71.3 \pm 5.1$ & $69.7 \pm 5.5$ & 0.032 \\
\hline Interventricular septum, mm & $20.2 \pm 4.9$ & $19.6 \pm 3.9$ & 0.265 \\
\hline Posterior LV wall, mm & $11.7 \pm 2.9$ & $12.2 \pm 2.9$ & 0.275 \\
\hline Maximal LV wall, mm & $22.4 \pm 4.9$ & $22.0 \pm 4.4$ & 0.528 \\
\hline SAM, \% & $100(94.3)$ & $94(88.7)$ & 0.217 \\
\hline LV outflow gradient, mm Hg & $78.8 \pm 28.2$ & $75.5 \pm 29.0$ & 0.412 \\
\hline
\end{tabular}

†, 185 patients had ambulatory ECG. BMI, body mass index; BSA, body surface area; ECG, electrocardiogram; HCM, hypertrophic cardiomyopathy; LV, left ventricular; LVEDD, left ventricular end-diastolic diameter; LVEF, left ventricular ejection fraction; NYHA, New York Heart Association; OCA, obstructive coronary artery; SAM, systolic anterior motion of mitral valve; SCD, sudden cardiac death. 